\title{
Experimental test of Water-Oil Emulsion Combustion
}

\author{
Askar Baubek ${ }^{1}$, Michael Zhumagulov, ${ }^{2,}$, Nurlan Kartjanov², Samal Sadykova ${ }^{3}$, and Muratbek Arpabekov ${ }^{1}$ \\ ${ }^{1}$ L.N. Gumilyov Eurasian National University, Dept. of Transport logistic, 2 Satpayev str, 010003 Nur-Sultan, Kazakhstan \\ ${ }^{2}$ L.N. Gumilyov Eurasian National University, Dept. of Power engineering, 2 Satpayev str, 010003 Nur-Sultan, Kazakhstan \\ ${ }^{3}$ Almaty University of Power Engineering and Telecommunications, , Dept. of Power engineering, 050013 Baytursynuly, 126/1, Almaty \\ c., Kazakstan
}

\begin{abstract}
This report dwells on the scientific study of the vortical device. The device is designed for efficient combustion of fossil fuels. Oil-water emulsion was taken as an example of alternative fuel. Although the functionality of the burner device is not limited to this fuel only. The innovative burner device under investigation is based on principal of vortical motion of fuel air mixture. There is a precombustion chamber specially designed, where inflammation and partial combustion of the swirling flow takes place. The article describes experiment on defining the geometric dimensioning of the burner discharge nozzle in relation to a combustion chamber. The position of the outlet nozzle inside of the burner is the main input parameter of this research. The authors analyzed the influence of this parameter on the pressure inside the combustion chamber and the electric load of the fan for the supplying combustion air. The position ranged from 0 to $120 \%$. The optimal position is at $50 \%$ of the total length of the burner chamber. The pressure graph has an extremum in this position, and the electric energy consumption by the fan changes less significantly with further deepening of the output nozzle than earlier. The Results are presented for cold (air blowing only) and hot experiment (burning). This experiment proved the need for the design of the inner part of the discharge nozzle, which is a design feature of the burner device for which the European patent was obtained. The patent link is presented in the list of references.
\end{abstract}

\section{Introduction}

Today, fuel-oil residual is used as a backup and sometimes primary fuel in most of the traditional thermal power plants in the world.

Fuel-oil residual is a type of oil fuel obtained from heavy refinery residues. Fuel-oil residual is, in fact, a mixture of hydrocarbons with a molecular mass of 4001000 , petroleum resins (with a molecular weight of 500-3000), carbenes, carboids, asphaltenes and other organic compounds.

Watering of fuel-oil residual occurs already at the stage of petroleum recovery -when it leaves the well together with the oilfield water and in oil desalting process.Further increase of moisture in residual fuel oil occurs when they are transported, prepared and stored [1].

In petroleum products, water can be contained as simple suspensions, then it is easily settled during storage, or in the form of a stable emulsion. The formation of stable oil emulsions leads to large financial losses. After separation of water from oil in sedimentation tanks and reservoirs, some part of oil is discharged together with water in the form of an emulsion and pollutes waste waters [1].

When fuel-oil residual of high viscosity is used in boiler houses as a liquid fuel, difficulties arise in preparing them for combustion. In most operating and projected industrial and heating boiler houses, the heating of fuel-oil residual delivered in railway tanks is provided by means of water vapor, which leads to wide range watering the fuel (from 5 to $20 \%$ ). Burning of watered fuel-oil residual without preliminary preparation causes disturbance of burning process (down to extinction of the flame) and reduces the economical efficiency of fuel-burning plants [1]. Creating a water-oil emulsion with a stable degree of homogeneity allows us to burn the mixture effectively without separating the fuel from the water [2-5]. The ecological performances are also increasing [6-11].

The purpose of this study is to justify the burning of fuel-oil residual in the form of a water-fuel emulsion replacing a long and sometimes costly settling and burning of fuel-oil residual as such.

For successful implementation of the task it is necessary to fulfill 2 conditions:

1. Homogenize the mixture of fuel and moisture in a special device (for example, a dispersant). This issue is widely studied in world practice, therefore it is not worthwhile to dwell on it in detail. The dispersant used by us is cavitating.

2. Effectively mix water-fuel emulsion with an oxidant (air) in a burner.

Further information in the article concerns the second condition.

* Corresponding author: zhmg 9@ mail.ru 
Fuel atomizeris used for the combustion of pure fueloil residual. Fuel oil atomization in the combustion chamber is carried out by the induction steam or the pressure of the fuel itself. It is common knowledge that such a design is ineffective for burning water-oil emulsion. The main reason for low-efficiency combustion is the poor contact of the fuel with the oxidant.

The basis of the combustion process is the aerodynamic structure of the gas-air flow, in where the combustion process takes place $[12,13]$. The role of the aerodynamic structure of the flow is to ensure a perfect mixture of fuel and oxidizer, without which neither the intensity of the combustion process nor the completeness of the heat release can be achieved.

A series of actions were taken at L.N.Gumilyev Eurasian National University (Astana, Kazakhstan), resulting in creation of a vortex combustion chamber (Figure 1) with a tangential fuel and air supply [14, 15]. Vortex principle organizing fuel combustion is used in the device. The burner device has a horizontal cylindrical shape and a nozzle. After the adjustment of the vortex combustion chamber at the pilot industrial unit, it was supposed to be installed on the boiler installation. The installation has advantages over all known analogs due to the part of the exhaust nozzle extending inside. The proposed burner device allows the stable burning of a water-oil emulsion with a moisture content of up to $30 \%$ due to intensive mixing of fuel and air. The installation has advantages over all known analogs due to the part of the discharge nozzle extending inside. Patent was received for this device for the utility model of the Russian Federation No. 128284 dated June 21, 2012 for the "Device for burning fuel" [14] and European patent EP No. 2684700 of September 24, 2016 [15].
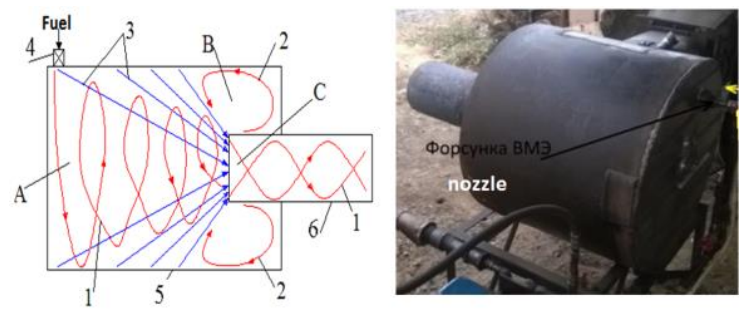

Fig. 1. Gas flow in vortex burner: 1- eddy motion of combustion products nozzle end, 2 - reverse current, 3 -flame vector in combustion chamber; 4 - supply nozzle; 5 - main chamber; 6 - discharge nozzle. Zones $A, B, C$.

\section{Description of the burner}

The fuel and oxidizer is fed into combustion chamber 5 (Figure 1) tangentially via supply nozzle 4 . The fuel and oxidizer enter the burner separately in form of swirling jet, then mixing in the main chamber 5. As it moves, before meeting with the secondary air, pre-heating of aeromixture occurs due to mass transfer with drawn-in hot gas stream and irradiation by burning torch, leading to oxidative reactions and ignition of the fuel particles. Mixing effect peak occurs at the inlet section of discharge nozzle 6 . Initiation of combustion begins in the combustion chamber 5 , coming with heat treatment of the fuel, the release of volatile fractions, partial coking etc. Complete and incomplete combustion products, twisting, form a stable vortex flow (Figure 1 shows the path of the gases with lines 1). Under the influence of the vortex gas movement discharge is formed in the axial zone A, and in zone B - reverse currents of 2 combustion products. 2, Reverse currents create overpressure in zone B, whereby the pressure in zone $\mathrm{B}$ exceeds the zone $\mathrm{C}$. As a result, flame vector 3 will be directed to the zone C. Thus, vortex flow in the combustion chamber take the form of tornado-shaped vortex with a constriction vortex radius as the gas goes to the outlet section. It should also be noted that the main vortex flow of variable section (mini tornado) practically does not touch the precombustor casing. Due to the part of the discharge nozzle extending inside the friction occurs in contact area of the main vortex of zone A with the reverse currents of zone $B$, as a result of which the overheating of the prechamber was almost completely eliminated.

In addition, an innovative cyclone-vortex burner is simple to manufacture and reliable in operation and modernization of the boiler does not require a significant investment.

\section{Laboratory tests}

The researches carried out had two stages: laboratory tests and mathematical modeling of aerodynamic flows. The present article describes the first stage. Initially, the research team set out the aim to explain experimentally the need of embedding discharge nozzle into the chamber. For this purpose, an experimental burner was assembled (Figure 2,3) with a variable position of part of the exhaust nozzle 2 extending inside. In the combustion chamber along the flow of the fuel-air medium, nozzles are installed for measuring pressure in three (I, II, III) points, apart from the discharge nozzle. The pressure value was determined by a U-shaped manometer. Current consumption of the installation was determined at the same time, upon readings of the ammeter connected to the power supply of the air fan motor, The value of the current consumed can characterize the total load on the fan, due to the aerodynamic resistance of the main path of the burner device.

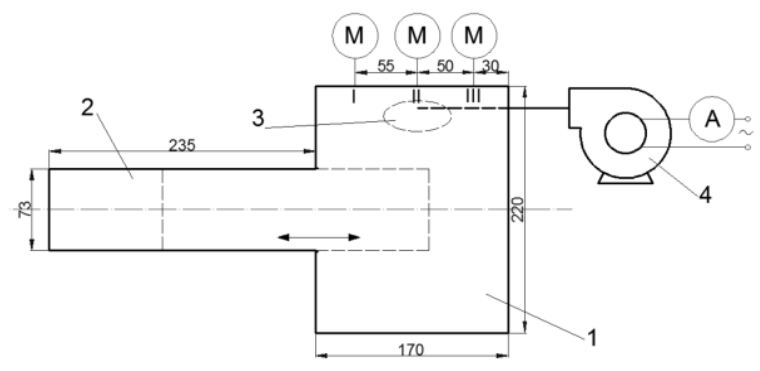

Fig. 2. Schematic layout of an experimental burner: the main chamber; 2 - discharge nozzle (mobile); 3 - inlet nozzle (for fuel and oxidizer supply); 4 - fan (for the supply of oxidizer); M-U-shaped manometers at points I, II, III; A-ammeter. 


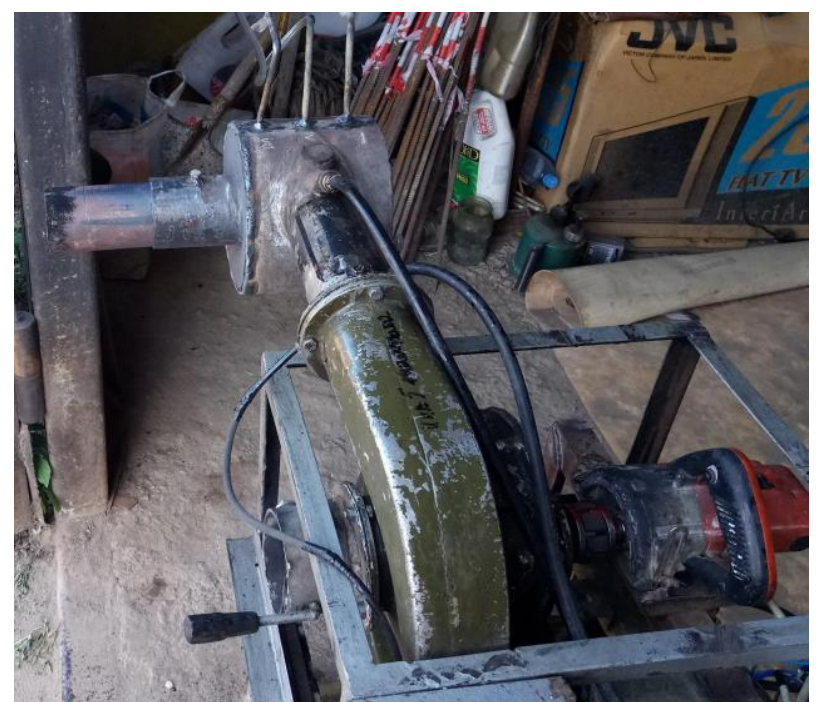

Fig. 3. Experimental burner with a mobile discharge nozzle.

The experiment was carried out in two stages:

1. «Cold» blow off of the combustion chamberwithout supply and burning of the fuel. Only air supply.

2. «Warm» stage - simultaneous supply of fuel and air with subsequent combustion and achievement of a stable flame.

During the experiment, the position of the projecting part of the discharge nozzle 2 gradually changed. The step of changing the position of the projecting part was $20 \mathrm{~mm}$. The first measurements were made in the absence of a nozzle part extending inside the furnace, which corresponds to the widely known analogues [13]. Then, step by step, the protruding part was buried inside.

As a result, the following experimental dependences were obtained:

Figure 4 shows the experimental dependence of the pressure inside the main chamber at points I, II and III on the position of the discharge nozzle. Measurements were made with a "Cold" blow off. The initial position (without embedding) is characterized by minimum pressure values. The first extremum is at the depth of the nozzle of $60 \mathrm{~mm}$, which corresponds to the maximum pressure at all three points. Subsequent embedding of the nozzle inside the chamber positively affects the pressure and leads to its decrease. This goal was set by the research team. The increased pressure in the chamber results in increased loads (over-expenditure of energy) on the blowing mechanisms, and also is an indicator of the increase in aerodynamic resistance. The second extremum is $100 \mathrm{~mm}$. When analyzing figure 4 without comparing it with other figures in this article, it is obvious that embedding the nozzle beyond the $100 \mathrm{~mm}$ mark will not lead to a positive result.

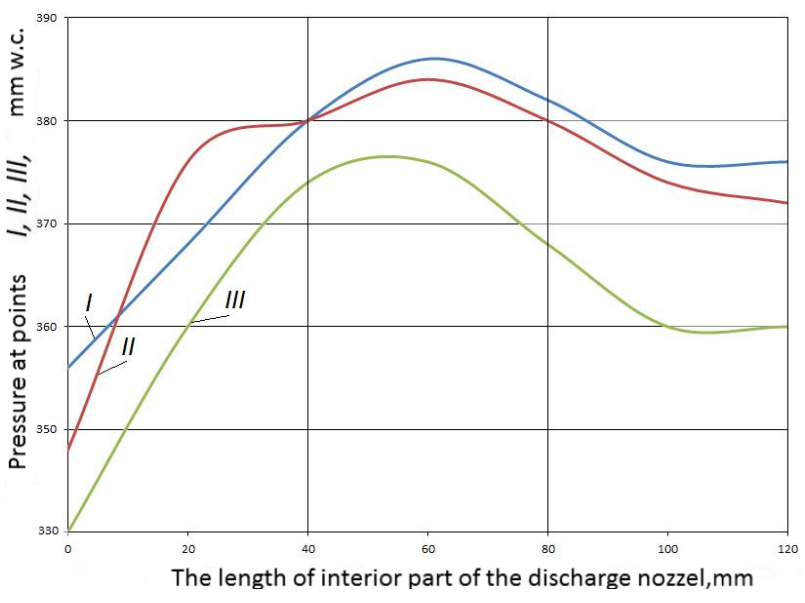

Fig. 4. The experimental dependence of the pressure inside the main chamber on the position of the discharge nozzle (cold stage).

Figure 5 shows the dependence of the fan current consumption on the position of the discharge nozzle. The level of power consumption on the fan is an indirect indicator of the aerodynamic resistance of the main path of the combustion chamber 1 (Figure 2). The curve clearly demonstrates a reduction in the current consumption with an increase in the degree of embedding of the discharge nozzle. The extremum of the curve lies at the level of the nozzle position $80 \mathrm{~mm}$ deep. Further embedding of the nozzle is not effective. Further, as noted above, the experiment was carried out under the conditions of the full-fledged functioning of the proposed burner device - the "warm" stage. The burner was supplied with diesel fuel, spark igniter was used for burning and similar measurements were carried out under real combustion conditions.

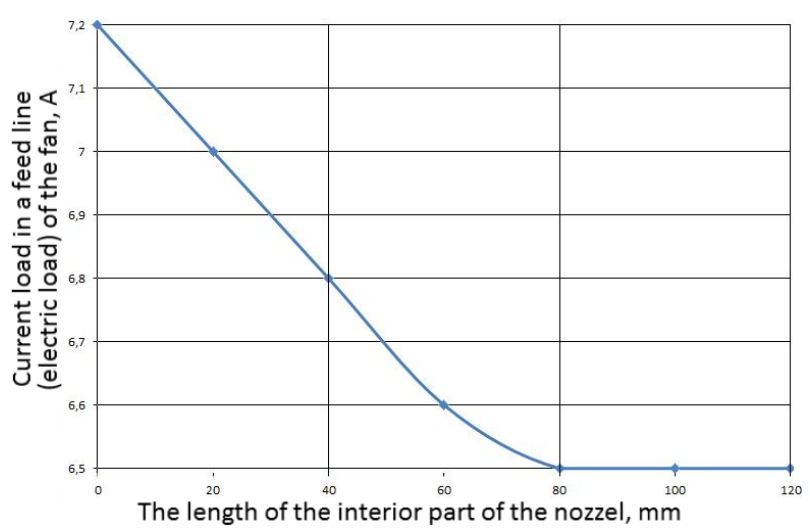

Fig. 5. The experimental dependence of the current consumption of the fan on the position of the discharge nozzle (cold stage).

Figure 6 is a graph of the pressure dependence within the main chamber on the position of the discharge nozzle in the conditions of a "warm" start of the burner device. In "warm" conditions, the initial position of the burner demonstrates a different behavior compared to cold blows. The maximum value of the pressure inside the chamber is measured in the absence of part of the discharge nozzle extending inside. With subsequent increase of extending part, the pressure drops. 


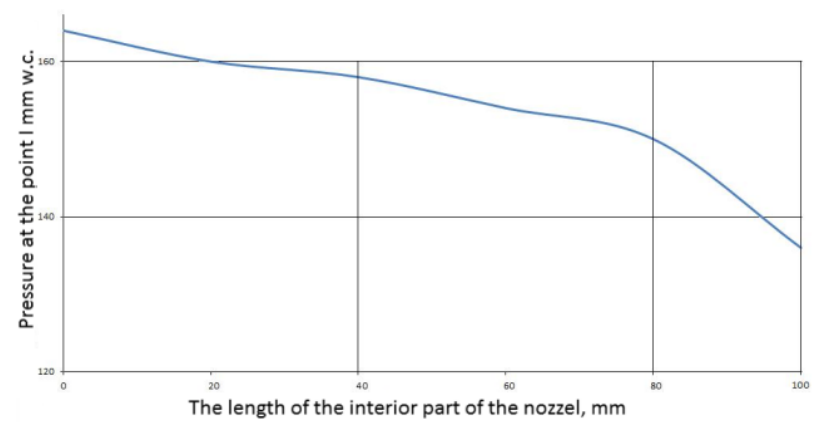

Fig. 6. The experimental dependence of the pressure inside the main chamber on the position of the discharge nozzle ("warm" stage).

Figure 7 shows the power consumption at the fan motor terminals. In general, the trend repeats the conditions of cold start (without burning fuel).

As a result, based on the results of the experiment, it is possible to draw a logical conclusion: the use of the discharge nozzle as a part extending inside of the main prechamber of combustion positively affects the aerodynamic characteristics of the fuel- air mixture. As the part extending inside increases, the pressure within the chamber decreases, alongside general reduction in energy consumption on the fan. For the experimental sample, the optimum position of the discharge nozzle inside the chamber is $80 \mathrm{~mm}$, which is $47 \%$ for a total length of a chamber in $170 \mathrm{~mm}$.

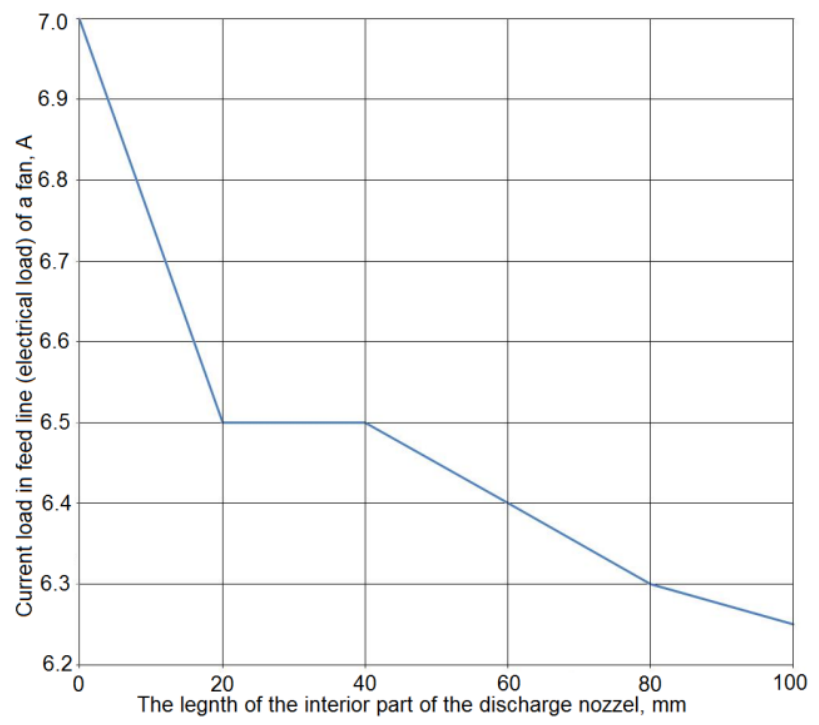

Fig. 7. The experimental dependence of the fan current consumption on the position of the discharge nozzle ("warm" stage).

At the next stage, it was decided to describe mathematically the aerodynamic flows in the proposed burner device in support of the experiment or to refute it. However, the format of this conference limits the number of pages in the report. Therefore, we are not provide simulation results here.

\section{Conclusion}

1. The necessity of changing the inner part of the discharge nozzle is substantiated. The recommended degree of embedding was $47-50 \%$ of the total length of the burner chamber;

2. Paten of Russian Federation and the European Patent Office was obtained for the structural characteristics of the combustion chamber (precombustor) and its elements;

3. The prototype of the burner showed perspective introduction of cyclone- vortex technology in boiler technology, as evidenced by the increase in their heating capacity, efficiency and a significant reduction in harmful emissions;

4. The minimum value of the horizontal coordinate for the outlet nozzle of small cross section was determined.

\section{References}

[1] G.F. Knorre, Combustion processes (Moscow: State Energy Publisher, 396, 1959)

[2] A.K. Hasannuddin, J.Y. Wira, R. Srithar, S. Sarah, M.I. Ahmad, S.A. Aizam, M.A.B. Aiman, M. Zahari, S. Watanabe, M.A. Azrin, S.S. Mohd. Effect of emulsion fuel on engine emissions-A review, Clean Technologies and Environmental Policy 18, 1 (2015)

[3] M.Y. Khan, Z.A.A. Karim, F.Y. Hagos, A.R.A. Aziz, I.M. Tan, Current Trends in Waterin-Diesel Emulsion as a Fuel, Scientific World Journal, 1-15 (2014)

[4] M.Z. Abu-Zaid, Performance of single cylinder direct injection diesel engine using water fuel emulsions, Energy Convers. Manag. 45, 697-705 (2004)

[5] A. Alahmer, J.Yamin, A. Sakhrieh, M.A. Hamdan, Engine performance using emulsified diesel fuel, Energy Convers Manag. 51, 1708-1713 (2010)

[6] R.K. Gopidesi, S.R. Premkartikkumar, Performance, emission and combustion analysis of diesel engine fuelled with emulsified biodiesel, Progress in Industrial Ecology - An International Journal 12, 1/2, 59-66 (2018)

[7] M. Kim, J. Oh, C. Lee, Study on Combustion and Emission Characteristics of Marine Diesel Oil and Water-In-Oil Emulsified Marine Diesel Oil, Energies 11, 1830 (2018)

[8] E.M. Afify, S.S. Korah, The effect of air charge temperature on performance, ignition delay and exhaust emissions of diesel engine using w/o emulsions as fuel, SAE Paper no. 870555 (1987)

[9] T.Xavier, M. Alain, NOx and PM emissions reduction on an automotive HSDI Diesel engine with water-in-diesel emulsion and EGR an experimental study, Fuel 31, 79-92 (2011) 
[10] Y.B. Zeldovich, Oxidation of nitrogen in combustion and explosion, Dokl Akad Nauk SSSR Seriya A51, 217-220 (1946)

[11] M. Tadashi, M. Yasushi, T. Minoru, M. Noboru, Experimental reduction of NOx, Smoke and BSFC in a Diesel engine using uniquely produced water $(0-80 \%)$ to fuel emulsion, SAE Paper no. 780224 (1978)

[12] K.A. Shtym, T.A. Solov'eva, Conversion of KVGM-100-150 boilers to cyclone-swirl burning of gas, Thermal Engineering 62, 3, 202-207 (2015)

[13] A.N. Shtym, Aerodynamics of cyclone-vortex chambers (Vladivostok: Far Eastern University Publisher, 200, (1984)

[14] A. Baubek, N. Baubek, Fuel combustion device, Patent of Russian Federation no. 128284 (2012)

[15] A. Baubek, N. Baubek, Device for burning fuel [Electronic resource], European Patent no. 2864700 (2016). Available at: https://data.epo.org/gpi/EP2864700B1-DEVICEFOR-BURNING-FUEL 